\title{
Psychological distress in cancer patients in Southern province of Sri Lanka
}

\author{
Weeratunga EB ${ }^{1}$, Senadheera $C^{1}$, Ekanayake $\mathbf{U}^{2}$ \\ ${ }^{I}$ Faculty of Medicine, University of Ruhuna, Galle, Sri Lanka. \\ ${ }^{2}$ Teaching Hospital, Karapitiya, Galle, Sri Lanka. \\ Correspondence: Dr. C Senadheera \\ e-mail: chandaniesenadheera@yahoo.co.uk
}

\begin{abstract}
Introduction: Stress in cancer patients directly affects the outcome of treatment. Aim of the study was to assess stress levels in cancer patients.

Methods: A sample of cancer patients $(\mathrm{N}=210)$ were assessed using an interviewer administered questionnaire and the General Health Questionnaire - 12 item version (GHQ-12).

Results: Whole sample had reported some level of distress; a large majority (65\%) had reported severe levels of distress (GHQ>20). The overall mean GHQ score (mGHQs) was $22.50(\mathrm{SD}=3.8)$. Severe distress level indicated by participants with different cancer types ranged from $57 \%$ of those with breast cancer to $84 \%$ with lung cancer. No significant differences were found in mGHQs of five cancer types $(\mathrm{p}=0.056)$. Half of the participants $(51 \%)$ had reported disabilities and their mGHQs $( \pm \mathrm{SD})$ was $23.79( \pm 4.30)$, while mGHQs $( \pm \mathrm{SD})$ of those without disability was $21.17( \pm 2.70)(\mathrm{p}<0.001)$. Among them, 49\% had two disabilities (mGHQs $-21.16 \pm 2.69), 27 \%$ had more than three disabilities (mGHQs - 24.82 \pm 4.62 ). Majority (64\%) earned monthly income of $<$ Rs. 10,000/- and their mGHQs was 23.19 while that of those who earned $>$ Rs. 10,000/- was 21.28 ( $<<0.001)$.
\end{abstract}

Conclusions: Lung cancer patients were severely distressed. Cancer patients with lower income and disabilities were reported to have higher distress. Psychosocial support services should prioritize patients experiencing disabilities and those who are from lower economic background.

Key Words: Cancer, psychological distress, disabilities, GHQ

\section{Introduction}

Worldwide, one in eight deaths are due to cancer; cancer causes more deaths than AIDS, Tuberculosis and Malaria combined (1). Cancer is the second leading cause of death in developed countries as well as in developing countries (1). An estimated 14.1 million new cancer cases and 8.2 million cancerrelated deaths occurred in 2012 worldwide, and incidence is higher among male gender (2). Risk of developing cancer is increased with increasing age (3). Cancer competes with heart diseases to become the leading cause of death in Sri Lanka $(3,4)$.
The patterns of diseases in Sri Lanka are somewhat similar to those in the developed countries. According to the National Cancer Control Programme (NCCP) in Sri Lanka, incidence and mortality rates of cancer patients have been increasing. In years 1985 and 2008, cancer incidence was 31.6 and 81.6 (crude rate per 100,000 populations) respectively (5).

When patients are diagnosed and living with a lifethreatening illness such as cancer, it causes high levels of stress. Stress is a common psychological problem among cancer patients and plays a major role in their lives. Distress develops during any 
phase of cancer, directly affects the outcome of the treatments (6). High incidence and prevalence of distress are common among cancer patients worldwide. One study reported higher levels of distress varying from $41 \%$ in patient with breast cancer to $23 \%$ in those with upper gastro-intestinal tract cancer (7). Overall prevalence of distress in cancer patients has been reported in the range of $35 \%$ $-38 \%$ (8). Lung, pancreatic, head and neck, Hodgkin's disease, and brain cancer patients were the most distressed (9).

International Classification of Functioning, Disability and Health (ICF) of World Health Organization (WHO) have defined the term 'disability' as impairments, activity limitation or participation restriction in daily activities (10). Physical disabilities/ functional limitations in daily activities, cancer related fatigue (CRF) are more common among cancer patients; and such conditions would severely affect the psychological well-being of terminally ill cancer patients, causing higher level of psychological distress $(6,11)$. As the best predictors of psychological distress, fatigue, metastasis, functional limitations (11) and fear of disease progression (7) were considered in the previous studies.

Impact of psychological distress on heterogeneous cancer patients in Sri Lanka is largely unknown. Although cancer victims a severe burden to the health system of the country, little attention has been paid. It was found that only a few studies have been done on stress in cancer patients (12). A study of a sample of 75 Breast Cancer (BC) patients reported that nearly $50 \%$ of the patients had experienced psychological distress during the period following their diagnosis (12).

There is a severe shortage of data pertaining to psychological profile of cancer patients in Sri Lanka. Such data are important to design effective strategies and psychosocial intervention to reduce stress and to improve psychological well-being of these patients who are the recipients of services from underresourced health system in Sri Lanka.

The aim of the present study was to assess the psychological distress in cancer patients treated at a tertiary care hospital in the Southern province of Sri Lanka. In addition, it was expected to identify the factors which influence the stress levels among cancer patients.

\section{Methods}

Two hundred and ten (210) randomly selected cancer patients who obtained the services from cancer unit in Teaching Hospital, Galle from May, 2013 to January, 2014 were studied using an interviewer administered questionnaire, bed head tickets and the diagnosis cards of the patients. Sinhala version of General Health Questionnaire- 12 item version (GHQ-12) $(13,14)$ was applied to measure short term psychological distress during previous few weeks.

First part of the questionnaire consisted of demographic data (age, sex, marital status, education, occupation, income levels, etc.) and clinical characteristics (cancer types, stage, time since diagnosis, disabilities, etc.) of the patients. Disabilities in daily functioning were assessed in 7 areas; walking, attending self-care, eating/drinking, talking, hearing, vision and involvement in household work. Subjects were asked to respond questions using Likert- type scale. In the second part, GHQ-12 scale was included and used to measure the stress levels among study population. GHQ, score of more than 15 was taken as evidence of distress while a score of more than 20 suggested severe problems and psychological distress.

Inclusion criteria of subjects included confirmed diagnoses as any type of cancer by the Consultant Oncologist, admitted to oncology wards in the above hospital, continuous treatment taken from oncology wards or oncology clinic for 6 month period. Patients aged 25 years or older who were able to understand Sinhala language were invited for the study. Those who provided informed consent with sufficient physical and mental stability were included. Patients who had surgical or medical complications or in critical state or end stage of cancer were excluded.

Ethical approval was granted from the Ethics Review Committee of the Faculty of Medicine, University of Ruhuna, Sri Lanka. Permission was obtained from the Director, relevant Consultants and Sisters In-Charges of the Teaching Hospital, Karapitiya, Sri Lanka. Patients were interviewed by the author in relevant oncology wards or follow-up clinics. Basic demographic details and other data were collected. 
All data were coded and entered into a database, analyzed using the Statistical Package of Social Science (SPSS) version 17.0. Basic descriptive statistics were calculated to describe the study sample and expressed as means and standard deviations. Bivariate statistical tests were used to identify associations between GHQ scores with demographic data and clinical characteristics. Independent samples t-test and one-way analysis of variance (ANOVA) were used to compare means of variables in two groups and three or more groups respectively. Pearson correlation coefficient was used to compare the relation between distress levels, cancer types, and socio-demographic parameters. All results were regarded as statically significant at $\mathrm{p}$ $<0.05$.

\section{Results}

Sample consisted of 97 breast (46\%), 32 oral (15\%), 32 colon (15\%), 25 lung (12\%) and 24 uterine cancer (11\%) patients. The mean age was 55 years (SD $=10.2$ ). Different types of disabilities affecting daily life such as eating/drinking (25\%), home activities (20\%), walking (19\%) were reported by the participants. Summary of socio-demographic details is given in Table 1.

Overall mean score of the GHQ scale was 22.50 $(\mathrm{SD}=3.8)$ and scores ranged from 16-35. The whole sample had reported some level of distress; a large majority $(65 \%)$ had reported severe levels of distress (GHQ>20).

Considering five cancer types, severe distress levels (GHQ $>20$ ) were found in $84 \%$ of lung cancer patients followed by $79 \%$ of uterine cancer patients, $69 \%$ of oral cancer, $59 \%$ of colon cancer and $57 \%$ of breast cancer patients.

The mean GHQ scores of patients with different cancer types were given in Table 2. No significant differences were found in mGHQs of the five cancer types $(p=0.056)$.

ut of studied socio-demographics and clinical characteristics of the participants, level of income and disabilities contributed to the stress level $(\mathrm{p}<0.001)$ (Table 3).
Table 1: Socio-demographic details of cancer patients

\begin{tabular}{|c|c|}
\hline Categories/variables & N $(\%)$ \\
\hline \multicolumn{2}{|l|}{ Age } \\
\hline$<55$ years & $113(54)$ \\
\hline$>55$ years & $97(46)$ \\
\hline \multicolumn{2}{|l|}{ Sex } \\
\hline Female & $149(70)$ \\
\hline Male & $61(30)$ \\
\hline \multicolumn{2}{|l|}{ Marital status } \\
\hline Married & $176(84)$ \\
\hline Unm arried / Single & $34(16)$ \\
\hline \multicolumn{2}{|l|}{ Educational status } \\
\hline Up to 10 years & $65(31)$ \\
\hline More than 10 years & $145(69)$ \\
\hline \multicolumn{2}{|l|}{ Employment status } \\
\hline Employed & $115(55)$ \\
\hline $\begin{array}{l}\text { Unemployed } \\
\qquad(\text { never worked/retired) }\end{array}$ & $95(45)$ \\
\hline \multicolumn{2}{|l|}{ Time since diagnosis } \\
\hline$<12$ months & $137(65)$ \\
\hline$>12$ months & $73(35)$ \\
\hline \multicolumn{2}{|l|}{ Self-reported disabilities } \\
\hline No & $103(49)$ \\
\hline Yes & $107(51)$ \\
\hline \multicolumn{2}{|l|}{ House hold income level } \\
\hline$<$ Rs. 10,000 per month & $134(64)$ \\
\hline$>$ Rs. 10,000 per month & $76(36)$ \\
\hline
\end{tabular}

$\mathrm{N}$ - Number of patients, \%- percentage of patients

Table 2: Mean GHQ scores by cancer types

\begin{tabular}{llll}
\hline Cancer type & $\begin{array}{l}\text { mGHQ } \\
\text { score }\end{array}$ & SD & p value \\
\hline Lung & 23.96 & 4.1 & \\
Oral & 23.28 & 4.4 & \\
Uterine & 23.21 & 3.3 & 0.056 \\
Breast & 21.95 & 3.7 & \\
Colon & 21.72 & 3.0 & \\
\hline mGHQs - mean GHQ scores, SD - standard \\
deviation, p value $<0.05$
\end{tabular}


Table 3: Influencing factors on stress levels of the study population

\begin{tabular}{llllll}
\hline Variable & $\mathbf{N}$ & $\mathbf{\%}$ & $\mathbf{m G H Q s}$ & SD & p value \\
\hline Income & & & & & \\
$\quad<$ Rs. 10,000 & 134 & 64 & 23.19 & 4.1 & $<0.001$ \\
$\quad$ Rs. 10,000 & 76 & 36 & 21.28 & 2.7 & \\
Disabilities & & & & & \\
$\quad$ Yes & 107 & 51 & 23.79 & 4.3 & $<0.001$ \\
$\quad$ No & 103 & 49 & 21.27 & 2.7 & \\
\hline
\end{tabular}

N- Number of patients, \%- percentage of patients, mGHQs- mean GHQ scores, SD- standard deviation, $\mathrm{p}$ value $<0.001$

Considering studied cancer patients who had disabilities, statistically significant differences in having disabilities were found among five cancer types $(p<0.001)$; oral, breast and uterine cancer patients had indicated significant differences in disabilities in multiple comparison (post hoc test).

Disabilities studied in this study were related to the type of cancer. Out of all oral cancer patients, $69 \%$ had reported disabilities in eating/drinking and $41 \%$ had reported disabilities in talking; $36 \%$ of lung cancer patients had reported disabilities in walking and $28 \%$ of them had reported disabilities in eating/ drinking and difficulties in doing home activities.
Among subjects with different cancer types, higher number of breast cancer patients had reported disabilities $(n=38,35 \%)$, followed by oral $(25 \%)$, colon (16\%), lung (16\%), and uterine $(8 \%)$ cancer patients (table 4). Irrespective of cancer type, all patients who reported disabilities had significantly high mGHQs compared to their counterparts who had not reported any disability except colon cancer patients (Table 4).

Further, mean stress scores were significantly related to the types of disabilities, number of disabilities and the different combinations of the disabilities. Table 5 illustrates mGHQs of patients with different types of disabilities.

Table 4: Comparison of mean GHQs of subjects with/without disabilities

\begin{tabular}{llllll}
\hline Cancer type & Disability & Number & mGHQs & SD & p value \\
\hline Breast & & & & & \\
& Yes & 38 & 23.39 & 4.6 & $0.006^{*}$ \\
Oral & No & 59 & 21.02 & 2.7 & \\
& & & & & \\
& Yes & 27 & 24.00 & 4.4 & $0.03^{*}$ \\
Colon & No & 05 & 19.40 & 1.9 & \\
& & & & & \\
& Yes & 17 & 22.12 & 3.4 & 0.43 \\
Lung & No & 15 & 21.17 & 2.5 & \\
& & & & & \\
\multirow{2}{*}{ Uterine } & Yes & 17 & 25.24 & 3.8 & $0.02^{*}$ \\
& No & 08 & 21.25 & 3.4 & \\
& & & & & \\
& Yes & 08 & 25.38 & 4.1 & $0.02^{*}$ \\
\hline
\end{tabular}

mGHQs- mean GHQ scores, SD- standard deviation, p value $<0.05, *$ significant differences among mGHQs of disabilities among cancer types 
Table 5: Mean GHQ score for different types of disabilities

\begin{tabular}{lcccc}
\hline Type & Disability & mGHQs & SD & p value \\
\hline Attending self-care & & & & \\
& Yes & 27.82 & 3.4 & $<0.001$ \\
& No & 22.21 & 6.2 & \\
Walking & & & & \\
& Yes & 25.46 & 4.4 & $<0.001$ \\
\multirow{2}{*}{ Home activities } & No & 21.82 & 3.3 & \\
& & & & \\
& Yes & 24.64 & 5.0 & $<0.001$ \\
Eating/ drinking & No & 21.96 & 3.2 & \\
& & & & $<0.001$ \\
& Yes & 24.60 & 4.3 & \\
\hline
\end{tabular}

\%- frequency of disability, mGHQs- mean GHQ scores, SD- standard deviation, p value $<0.001$

Comparing patients who had different numbers of disabilities (eg: two, three or more), $49 \%$ of the sample had reported two disabilities and $27 \%$ had reported more than three disabilities $(\mathrm{p}<0.001)$. A higher mGHQ score was reported by patients who had more than three disabilities $(24.82 \pm 4.62)$.

Stress levels were significantly associated with different combination of disabilities they have experienced. Among various combinations like walking - attending self-care, eating - attending selfcare etc., combination of talking - attending self-care had scored the highest mGHQ (32.00 \pm 1.4$)$ than other studied disabilities in this group of patients.

\section{Discussion}

All cancer patients in this study reported some level of distress; $65 \%$ found to have severe stress. Some western studies report the prevalence rate of stress in cancer patients in the range of $35 \%-38 \%(8,9)$. Compared to western samples, our study sample reported higher stress levels. Community study of cancer outpatients reported lower prevalence rate of stress $(24.5 \%)$ (15), compared to that of patients who were in large tertiary cancer center (37.8\%) (9). Another study has found prevalence of cancerrelated distress in a range of $24 \%-59 \%$ depending on the type of cancer, and higher levels of distress varied from $41 \%$ in breast cancer (BC) to $23 \%$ in upper gastro-intestinal (GI) tract cancer patients (7). In addition Gao et al. , (15) suggested that cancer type/site was the best predictor for psychological distress in various treatment settings.

Among five cancer types which we studied, severe psychological distress (GHQ score $\geqslant 20$ ) was found in $84 \%$ of lung cancer patients. Zabora et al. (8) had compared 14 cancer types and stress levels; indicating that lung cancer (LC) patients had scored the highest stress level (43.4\%) while gynecological cancer patients experienced lowest stress level (29.6\%). In the current study, the highest mean GHQ score was reported by lung cancer patients $(23.96 \pm 4.1)$ and the lowest mean stress score was reported by colon cancer $(21.72 \pm 3.0)$ patients. Other studies in the west also reported similar findings $(8,16)$. Among all cancer types of present study, BC patients had scored the lowest prevalence of stress (57\%), and it was still higher than the levels found in BC patients in Herschbach's study (41\%). In the same study prevalence of stress in GI cancer patients was lower $(23 \%)$ while in our study, the prevalence of stress was higher in colon cancer patients (59\%). Lung, pancreatic, head/ neck, Hodgkin's disease, and brain cancer patients were the most 
distressed patients when considered the cancer sites (9).

Majority of our sample reported lower monthly income, and they scored higher mGHQ. In other words psychological distress was higher in cancer victims who had lower income ( $<$ Rs. 10,000/-). Psychological distress of cancer patients was reported to be decreased with increase in income levels $(8,9,16)$. Unemployed BC women significantly had more stress; their stress can be lowered by employment (16). The reasons for having low stress in employed women might be related to the ability to overcome issues in a better manner as a result of having a wider social environment and self-confidence (16). However, there is evidence that most cancer survivors have suffered from significant work-related disabilities (17) which may affect work performance, resulting in low income or turn-out from the job, causing further increase in distress.

Different types of disabilities were reported by the participants of the present study. Irrespective of the cancer type, those experiencing disabilities have to depend on others to different extents. It may contribute to increase their stress levels further. Many western studies had reported similar findings on disabilities and stress levels in cancer patients; usually starting as discomfort or pain and severely affecting the psychological well-being of terminally ill cancer patients; due to worsening of physical functions and cancer-related fatigue $(6,11,18)$. A high prevalence of limitations in daily activities and/ or instrumental activities was reported by the elderly cancer patients (19). Considering LC patients in the present study, they had experienced various percentages of different types of disabilities and it may have led them to score the highest stress than others. Similarly in a study of women in USA who had lung cancer reported poor physical functioning, than women with breast cancer (20).

In Sri Lanka it is less known how cultural factors such as beliefs about cancer affect the patients psychologically. Discrepancies in accessibility of health care system to different sectors of population hinder early detection of cancer and also prevent getting proper treatment. As a result, cancer patients may develop higher levels of fear and anxiety when they are diagnosed with a cancer. In addition, socioeconomic burden also contributes to psychological distress. Chandwani and colleagues had revealed that failure in early detection and interventions lead to elevated levels of distress with a negative impact on cancer outcomes, cancer therapies, survival and disease progression, resulting a decrease in patients' quality of life, and increase in care costs (19). However, further research is recommended to screen stress among cancer patients routinely in local setting.

Results of the current study may not be applicable to whole country due to the selected single study setting. It was a limitation of this study.

\section{Conclusions}

In our study, psychological distress affects all cancer patients, and is a significant health problem. Stress levels in cancer patients were influenced by some socio-economic factors, type of cancer, type of disability, number of disabilities, and combination of disabilities they have experienced. Those who had lower income and had disabilities were severely stressed.

Lung cancer patients were severely distressed compared to those with other types of cancers. Psychosocial factors contributing to psychological distress in cancer patients need to be studied further. Psychosocial support services should target more vulnerable patients experiencing disabilities and those who are from lower economic background. Studies in other countries emphasize the need for maintaining patients' daily activities and providing support to cope with cancer-related physical disabilities and emotional distresses (24). Therefore special attention must be paid to detect cancer patients with high level of stress and to address their psychosocial needs appropriately.

\section{Acknowledgment}

The author wishes to thank all participants; consultants who gave their permission to conduct the study at the cancer unit, and administrative staff and health care professionals at Teaching Hospital, Karapitiya; Coordinator and staff members of the Nursing Degree Programme, Faculty of Medicine, and University of Ruhuna, The author wishes to express her sincere gratitude to Prof. Bilesha Perera, Department of Community Medicine, Faculty of Medicine, University of Ruhuna for valuable 
guidance and encouragement provided being the principal supervisor. The study was funded by the Faculty Research Grants 2013- Faculty of Medicine, University of Ruhuna.

An oral presentation based on this study was done on 02.02.2016 at International Multidisciplinary Research Conference 2016 in Colombo. Title of abstract was "Psychological distress in cancer patients in Southern province of Sri Lanka, (Multidisciplinary Research 2016- ISBN 978-09939889-8-1).

\section{References}

1. American Cancer Society. Cancer facts and figures, $2^{\text {nd }}$ ed. Atlanta.American Cancer Society Inc, 2011.

2. IARC- WHO. Latest world cancer statistics- GLOBOCAN 2012. (Press release) 12 December 2013.

3. Kissane DW, Maj M, Sartorius N. Depression and Cancer.eds. John Wiley \& Sons, World Psychiatric Association, 2011.

4. Medical Statistical Unit. Annual health bulletin. Sri Lanka: Ministry of Health, 2012.

5. National Cancer Control Programme. Cancer Incidence Data. Sri Lanka: Ministry of Health \& Indigenous Medicine, 2008.

6. Taylor SE. Health Psychology. $6^{\text {th }}$ ed. McGraw Hill: University of California, 2006.

7. Herschbach P, Keller M, Knight L et al. Psychological problems of cancer patients: a cancer distress screening with a cancer-specific questionnaire. British Journal of Cancer 2004; 91: 504-11.

8. Zabora J, Brintzenhofeszo K, Curbow B, et al. The prevalence of psychological distress by cancer site. PsychoOncology2001; 10: 19-28.

9. Carlson LE, Angen M, Cullum J, et al. (2004). High levels of untreated distress and fatigue in cancer patients. British Journal of Cancer2004; 90: 2297-2304.
10. International Classification of Functioning, Disability and Health. $2^{\text {nd }}$ ed. Geneva: World Health Organization, 2001.

11. Valdes-Stauber J, Vietz E, Kilian R. The impact of clinical conditions and social factors on the psychological distress of cancer patients: an explorative study at a consultation and liaison service in a rural general hospital. BMC Psychiatry 2013; 13: 226.

12. Mudduwa L, Punchihewa G. Psychological impact of breast cancer: a study done in a Sri Lankan setting. Galle Medical Journal2011; 16: 16-21.

13. Goldberg D. Identifying psychiatric illness among general medical patients. British Medical Journal1985; 291:161-62.

14. De Silva N, Samarasinghe D. Acceptance of psychiatric screening-questionnaire by general practice attenders. Ceylon Medical Journal1990; 35: 105-08.

15. Gao W, Bennet MI, Stark D et al. Psychological distress in cancer from survivorship to end of life care: prevalence, associated factors and clinical implications. Eur J Cancer $2010 ; 9$.

16. Ogce F, Okan S, Baltalarli B. Psychosocial stressors, social support and socio-demographic variables as determinants of quality of life of Turkish breast cancer patients. Asian Pacific Journal of Cancer Prevention2007; 8: 77-82.

17. Oberst K, Bradley CJ, Gardiner JC, et al. Work task disability in employed breast and prostate cancer patients. $J$ Cancer Surviv 2010; 4: 322-30.

18. Serraino D, Fratino L, Zagonel V, et al. Prevalence of functional disability among elderly patients with cancer. Critical Review in Oncology/Hematology2001; 39: 269-73.

19. Chandwani KD, Ryan JL, Peppone LJ, et al. Cancer-related Stress and complementary andalternative medicine: a review. Evidence-Based Complementary and Alternative Medicine2012; 15.

20. Fang CY, Manne SL, Pape SJ. 2001. Functional impairment, marital quality, and patient psychological distress as predictors of psychological distress among cancer patients' spouses. Health Psychology 2001; 20: 452-47. 